\title{
Balanced Quantum-Inspired Evolutionary Algorithm for Multiple Knapsack Problem
}

\author{
C. Patvardhan, Sulabh Bansal* \\ Faculty of Engineering, Dayalbagh Educational Institute, Dayalbagh, Agra. 282005
}

\begin{abstract}
Anand Srivastav
Christian-Albrechts-Universität zu Kiel, Institut für Informatik, Christian-Albrechts-Platz 4, 24118 Kiel, Germany
\end{abstract}

\begin{abstract}
Multiple Knapsack Problem, a generalization of more popular 0/1 Knapsack Problem, is NP-hard and considered harder than simple Knapsack Problem. 0/1 Multiple Knapsack Problem has many applications in disciplines related to computer science and operations research. Quantum Inspired Evolutionary Algorithms (QIEAs), a subclass of Evolutionary algorithms, are considered effective to solve difficult problems particularly NP-hard combinatorial optimization problems. A hybrid QIEA is presented for multiple knapsack problem which incorporates several features for better balance between exploration and exploitation. The proposed QIEA, dubbed QIEA-M KP, provides significantly improved performance over simple QIEA from both the perspectives viz., the quality of solutions and computational effort required to reach the best solution. QIEA-MKP is also able to provide the solutions that are better than those obtained using a well known heuristic alone.
\end{abstract}

Index Terms-Hybrid Evolutionary Algorithm, Quantum Inspired Evolutionary Algorithm, Combinatorial Optimization, Multiple Knapsack Problem

\section{INTRODUCTION}

0-1 Multiple Knapsack Problem (MKP) is a generalization of the standard 0-1 knapsack problem (KP) where multiple knapsacks are considered to be filled instead of one. The MKP problem is strongly NPcomplete and no FPTAS is possible for MKP [1].

Evolutionary Algorithms (EAs) refer to a class of population based search technique used to obtain good solutions for hard optimization problems in general. Individuals in population map to solutions of the problem. The new generations are evolved with an objective to improve quality of solutions. Evolution of a new population involves application of various operators on members of existing population. Quantum-Inspired Evolutionary Algorithms (QIEAs) is subclass of EAs where the representation of individuals and operators involved in generation of new individuals are both designed based on the concept of Quantum Computing.

Various forms of QIEAs have been used to solve a variety of difficult problems for example $[2,3,4,5,6,7,8,9,10,11]$. QIEAs have been observed as a powerful tool because of their better representation power [12,13], EDA style of functioning [14,15], flexibility necessary for the inclusion of features appropriate for a given problem towards delivering better search performance [15], inherent quality of starting with exploration and gradually shifting towards the exploitation [13].

QIEA in itself only provides a very broad framework. QIEA, just as other EAs, suffers from several limitations. Small qubit rotations lead to slow convergence while large qubit rotations may cause the algorithm to miss a good solution completely. Inclusion of features promoting faster convergence may cause the algorith $m$ to get stuck in local optima. Slow convergence limits the problem sizes that can be tackled using QIEAs. Implementation of QIEAs, therefore, is more an art. Any attempt to solve a difficult problem has to use this framework judiciously and include features suited to the particular problem in order to get the desired performance. The objective in any attempted implementation of QIEA is to balance exploration and exploitation thus achieving convergence to optimal or near optimal solutions without requiring prohibitively large computation even for larger problem sizes.

Hybridizing the population based meta-heuristic search technique with heuristics algorithms available for particular problem is an approach that is popular since last few decades to solve difficult optimization problems [16]. The main motivation behind the hybridization of different algorithms is to exploit the complementary character of different optimization strategies. Hybrid meta-heuristic algorithms try to establish a balance between exploration and exploitation of the search space.

The population based search approaches are good at exploration of the search space and identifying areas with high quality solutions while they are not so effective in exploitation of these high quality areas. On the other hand the strength of local search is the capability of quickly finding better solutions in the vicinity of starting solutions. Generally the heuristics available for optimization problems are based on some kind of local search on an initial solution. Thus in such algorithms, which hybridize a meta-heuristic with a heuristic, the meta-heuristic approach can guide the global search and problem domain specific heuristic can help searching locally around the good solutions found from global perspective. 
Table 1. Modifications applied on QIEA's for KP in the literature

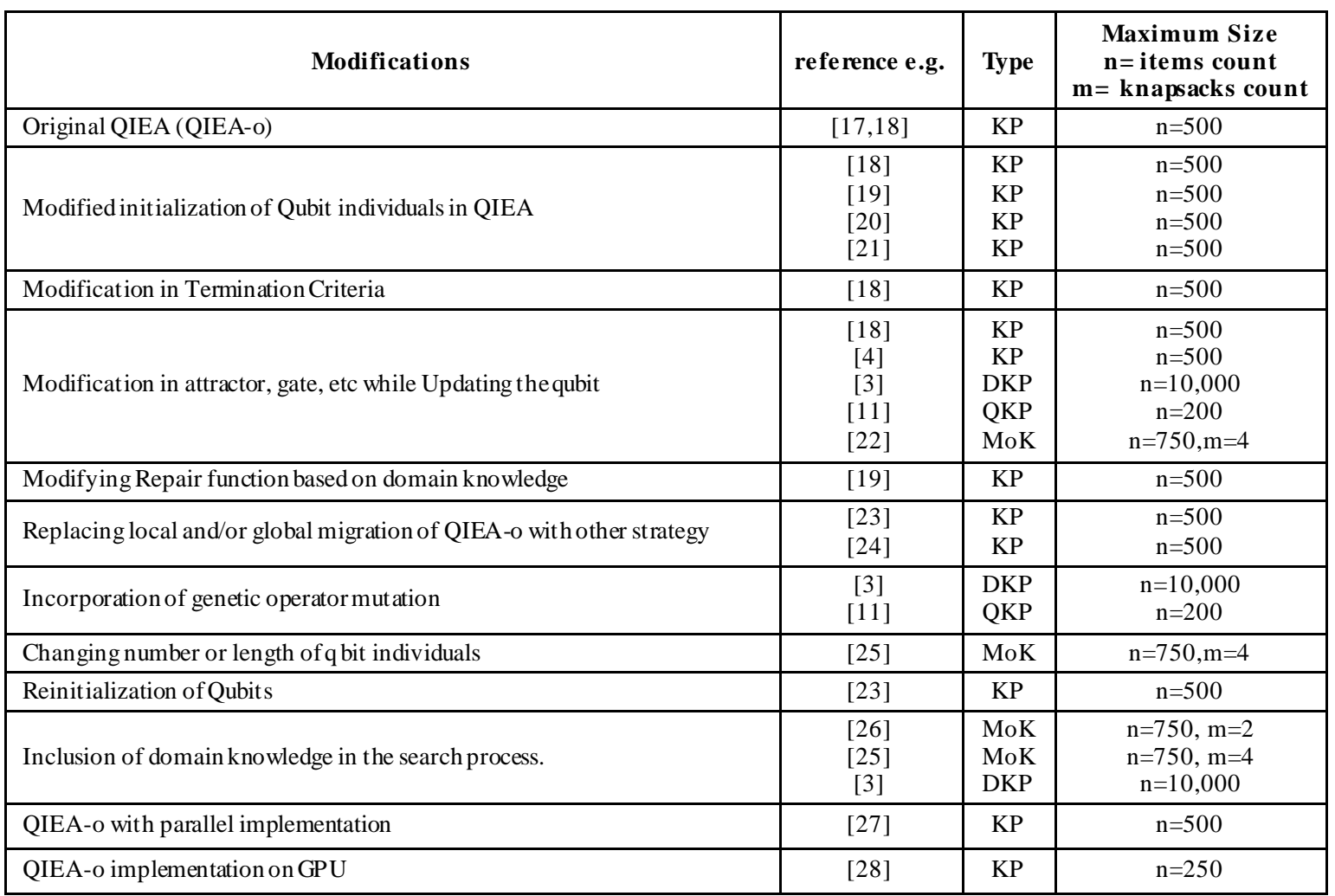

$* \mathrm{KP}=0 / 1$ Knapsack Problem, MoK = Multi-objective Knapsack Problem, QKP = Quadratic Knapsack Problem, DKP= Difficult 0/1 Knapsack problems

Thus, QIEAs with various modifications have been applied to different variants of popular combinatorial problem called Knapsack Problem (KP). Table 1 lis ts the modifications applied to QIEAs with the example existing in literature.

In this paper an attempt is made to design a hybrid QIEA balanced in its power to exploit and explore the search space in order to solve instances of MKP. The population based meta-heuristic QIEA is hybridised with an existing heuristic for MKP known as MTHM [29] and some additional features of population based search are judiciously incorporated in order to solve randomly generated instances of MKP. This is first attempt to solve MKP using such a meta-heuristic technique.

The rest of the paper is organized as follows. A brief description of MKP with a survey of approaches existing in literature for MKP is presented in section II. A brief conceptual description of a typical QIEA is given in section III. The MTHM heuristic used for hybridizing QIEA is discussed in section IV. In section V the QIEA framework used here and the proposed QIEA-MKP is explained in detail. Computational performance of QIEA-MKP is presented in section VI. Conclusions are presented in section VII.

\section{MULTIPLE KNAPSACK PROBLEM (MKP)}

Given a set of $n$ items with their profits $p_{j}$ and weights $\mathrm{w}_{\mathrm{j}}, \mathrm{j} \in\{1, \ldots, \mathrm{n}\}$, and $\mathrm{m}$ knapsacks with capacities $c_{i}, i \in\{1, \ldots, m\}$, the MKP is to select a subset of items to fill given $m$ knapsacks such that the total profit is maximized and sum of weights in each knapsack $i$ doesn't exceed the capacity $c_{i}$. $\operatorname{maximize}: \sum_{\mathrm{i}=1}^{\mathrm{m}} \sum_{\mathrm{j}=1}^{\mathrm{n}} \mathrm{p}_{\mathrm{j}} \mathrm{x}_{\mathrm{ij}}$ subject to : $\sum_{\mathrm{j}=1}^{\mathrm{n}} \mathrm{w}_{\mathrm{j}} \mathrm{x}_{\mathrm{ij}} \leq \mathrm{c}_{\mathrm{i}}, \mathrm{i} \in\{1, \ldots, \mathrm{m}\}$,

$$
\sum_{\mathrm{i}=1}^{\mathrm{m}} \mathrm{x}_{\mathrm{ij}} \leq 1, \mathrm{j} \in\{1, \ldots, \mathrm{n}\} \text {, }
$$

$\mathrm{x}_{\mathrm{ij}} \in\{0,1\}, \forall \mathrm{i} \in\{1, \ldots, \mathrm{m}\}, \forall \mathrm{j} \in\{1, \ldots, \mathrm{n}\}$,

where $x_{i j}=1$ if item $\mathrm{j}$ is assigned to knapsack $\mathrm{i}, \mathrm{x}_{\mathrm{ij}}=0$ otherwise and coefficients $\mathrm{p}_{\mathrm{j}}, \mathrm{w}_{\mathrm{j}}$ and $\mathrm{c}_{\mathrm{i}}$ are positive integers.

In order to avoid any trivial case, the following assumptions are made

1. Every item has a chance to be placed at least in largest knapsack:

$$
\max _{\mathrm{j} \in \mathrm{N}} \mathrm{w}_{\mathrm{j}} \leq \max _{\mathrm{i} \in\{1, \ldots, \mathrm{m}\}} \mathrm{c}_{\mathrm{i}} .
$$

2. The smallest knapsack can be filled at least by the smallest item:

$$
\min _{\mathrm{i} \in\{1, \ldots, \mathrm{m}\}} \mathrm{c}_{\mathrm{i}} \leq \min _{\mathrm{j} \in \mathrm{N}} \mathrm{W}_{\mathrm{j}} .
$$

3. There is no knapsack which can be filled with all items of $\mathrm{N}$ :

$$
\sum_{\mathrm{j}=1}^{\mathrm{n}} \mathrm{w}_{\mathrm{j}} \geq \mathrm{c}_{\mathrm{i}}, \forall \mathrm{i} \in\{1, \ldots, \mathrm{m}\} .
$$

The subset sum variant of MKP having $\mathrm{p}_{\mathrm{j}}=\mathrm{w}_{\mathrm{j}}, \mathrm{j} \in\{1, \ldots, \mathrm{n}\}$, is known as multiple subset sum problem (MSSP).

MKP has many applications in fields related to computer science and operations research. An application 
is seen when scheduling jobs on processors where some machines unavailable for a fixed duration or some high propriety jobs are pre-assigned to processors [30]. A real world application of MKP is the problem of cargo loading where some containers need to be chosen from a set of $n$ containers to be loaded in $\mathrm{m}$ vessels with different loading capacities for the shipment of the containers [31]. Another real world problem for MSSP is mentioned in [32] from a company producing objects of marble. The company receives $\mathrm{m}$ marble slabs, of uniform size, from a quarry. Each product, that company produces, requires a piece from marble slab having a specified length. Out of the list of products to be prepared some products need to be selected and cut from the slabs so that the total amount of wasted marble is minimized. This problem is modelled as MSSP with slabs corresponding to knapsacks and the lengths corres ponding to the weights.

Several attempts to solve the MKP exist e.g. [33,29,34] etc. Hung \& Fisk [33] presented a depth first branch and bound algorith $\mathrm{m}$ where the upper bounds were derived by Lagrangian relaxation. Martello \& Toth [29] proposed a different branch and bound algorithm where constraint $\sum_{\mathrm{i}=1}^{\mathrm{m}} \mathrm{x}_{\mathrm{ij}} \leq 1$ is omitted at each decision node and the branching item was chosen as an item which had been packed in $\mathrm{k}>1$ knapsacks of the relaxed problem. In a later work Martello \& Toth [34] proposed a bound and bound algorith $\mathrm{m}$ where at each node of the branching tree both the upper bound and lower bound are derived.

Some approximation algorithms also exist for MKP. Kellerer [35] presented the PTAS for MKP with identical capacities. Chekuri \& Khanna [1] generalized it and presented the PTAS for MKP. He also discussed MKP as a special case of the generalized assignment problem (GAP). GAP is APX-hard and only a 2-approximation exists. It is also shown that no FPTAS is possible for MKP. A PTAS containing the two steps, guessing the items as first and packing them as second, is presented subsequently in [1]. The EPTAS is designed based on the LP relaxation of the MKP by Jansen [36,37]. Jansen [37] presented a faster version of the algorithm designed in [36]. These approximation schemes claim to provide solution having approximation ratio of $1 / \epsilon$. The algorith $m$ is shown to take polynomial time with respect to size of the problem but is exponential with respect to the $1 / \epsilon$.

\section{QUANTUM INSPIRED EVOLUTIONARY ALGORITHM (QIEA)}

The QIEAs introduced in [17] are population-based stochastic evolutionary algorithms. They use the qubit, a vector, to represent the probabilistic state of individual. Each qubit is represented as $\mathrm{q}_{\mathrm{i}}=\left[\begin{array}{c}\alpha_{i} \\ \beta_{i}\end{array}\right], \alpha_{i}, \beta_{\mathrm{i}}$ are complex numbers so that $\left|\alpha_{\mathrm{i}}\right|^{2}$ is the probability of state being 1 and $\left|\beta_{\mathrm{i}}\right|^{2}$ is the probability of state being 0 such that $\left|\alpha_{i}\right|^{2}+\left|\beta_{i}\right|^{2}=1$. For the purpose of QIEAs, $\alpha_{i}$ and $\beta_{i}$ are assumed to be real. Thus, a qubit string with $\mathrm{n}$ bits represents a superposition of $2^{\mathrm{n}}$ binary states and provides an extremely compact representation of entire space.

The process of generating binary strings from the qubit string, Q, is known as observation. To observe the qubit string $\mathrm{Q}$, a string consisting of the same number of random numbers between 0 and $1(R)$ is generated. The element $P_{i}$ is set to 0 if $R_{i}$ is less than square of $Q_{i}$ and 1 otherwise. In each of the iterations, several solution strings are generated from $\mathrm{Q}$ by observation as given above and their fitness values are computed. The solution with best fitness is identified. The updating process moves the elements of $\mathrm{Q}$ towards the best solution slightly such that there is a higher probability of generation of solution strings, which are similar to best solution, in subsequent iterations. A quantum gate is utilised for this purpose [17].

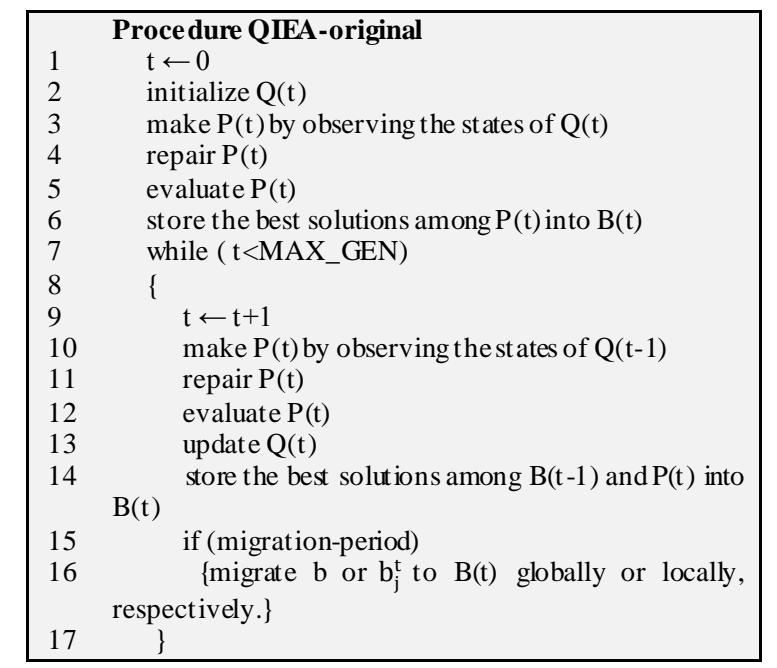

Fig. 1. Quantum Inspired Evolutionary Algorithm

One such gate, used by the QIEAs presented in this work, is the Rotation Gate, which updates the qubits as follows:

$$
\left[\begin{array}{l}
\alpha_{\mathrm{i}}^{\mathrm{t}+1} \\
\beta_{\mathrm{i}}^{\mathrm{t}+1}
\end{array}\right]=\left[\begin{array}{ll}
\cos \left(\Delta \theta_{\mathrm{i}}\right) & -\sin \left(\Delta \theta_{\mathrm{i}}\right) \\
\sin \left(\Delta \theta_{\mathrm{i}}\right) & \cos \left(\Delta \theta_{\mathrm{i}}\right)
\end{array}\right]\left[\begin{array}{l}
\alpha_{\mathrm{i}}^{\mathrm{t}} \\
\beta_{\mathrm{i}}^{\mathrm{t}}
\end{array}\right]
$$

where, $\alpha_{i}^{t+1}$ and $\beta_{i}^{t+1}$ denote probabilities for $i^{\text {th }}$ qubit in $(\mathrm{t}+1)^{\text {th }}$ iteration and $\Delta \theta_{\mathrm{i}}$ is equivalent to the step size in typical iterative algorithms in the sense that it defines the rate of movement towards the currently perceived optimum.

The above description outlines the basic elements of QIEA. Observing a qubit string ' $n$ ' times yields ' $n$ ' different solutions because of the probabilities involved. The fitness of these is computed and the qubit string $Q$ is updated towards higher probability of producing strings similar to the one with highest fitness. This sequence of steps continues; these ideas can be easily generalised to work with multiple qubit strings.

Pseudo-code for the QIEA orig inally proposed by Han \& Kim [17] is presented in fig 1 . 


\section{MTHM HEURISTIC FOR MKP}

Martello \& Toth [29] gave a heuristic algorith m, called MTHM, for solving the MKP. This algorithm consists of three phases as described in the following. The details are available in [38].

1. Initial feasible solution is obtained in the first phase of MTHM by applying the Greedy algorithm to the first knapsack; a set of remaining items is obtained, then the same procedure is applied for the second knapsack; this is continued till the $\mathrm{m}^{\text {th }}$ knapsack.

2. The initial solution is improved during the second phase by swapping every pairs of items assigned to different knapsacks and insert a new item such that the total profit is increased.

3. Each selected item is tried to be replaced by one or more remaining items during the last if possible so that the total profit sum is increased.

The MTHM heuristic has the advantage that some items can be exchanged from a knapsack to another or excluded from the solution set so that total profit increases, which can lead to an efficient and fast solution when the solution given by the first phase is good. Moreover they can be applied to any feasible solution effectively. The main drawback of MTHM heuristic is that it considers only the exchanges between a pairs of items and not the combinations of items.

Various phases of this heuristic can be introduced in QIEA to improve the quality of solutions found. In section $\mathrm{V}$ use of these to improve the performance of QIEA-MKP is examined in detail. The first phase of this heuristic is used to repair the infeasible solutions generated by collapse operation of QIEA. The second and third phases are applied as local search technique on the solutions improved from global perspective during the iterations of QIEA.

\section{QIEA-MKP}

In this work a modified QIEA framework is used as the starting point. In a QIEA the update operator is used to gradually modify the qubit individuals using an attractor such that it can generate solutions more similar to the attractor. QIEA as described by Han \& Kim [17] maintains a population of local best individuals corresponding to the population of qubit individuals besides the global best individual generated so far. Thus, the qubit individuals evolve according to different local best solutions but same global best solution. The update operator is applied either locally or g lobally, at local level the corresponding local best individual is used as an attractor and at global level the global best individual is used as an attractor. The global and/or local best solutions are replaced if found worse than the new solutions generated using a qubit individual. These new solutions are used as attractors subsequently. Here instead of generating single solution, a qubit individual generates multiple individuals every time before comparing those with stored best individuals generated so far such that only the best out of all these multiple solutions generated is actually compared with local or global best individuals available. This helps in exploitation of the areas of solution space represented by the particular qubit individuals more intensively before rotating them towards an attractor. The qubit individual is rotated towards the local best solution more often than the global best solution. Such an arrangement establishes the balanced capability to explore and exploit simultaneously which is a foremost requirement for any meta-heuristic implementation.

The above description provides a broad framework of QIEA with scope for enhancements for rapid solution of a specific problem. This frame work is used as the starting point here which has been enhanced with several features. The modified algorithm designed for MKP is named as QIEA-MKP. The improvements brought about in QIEA-MKP are as follows.

(i) The items are sorted in order of decreasing profit by weight ratio to improve the domain knowledge in the following

a. Initializing the Qubit Individuals so that they generate better solutions.

b. Modifying the repair function to improve quality of solutions.

(ii) Improving the local best solutions using local search

(iii) Mutation of solutions when they appear to be stuck in a local optimum

(iv) Re-initialization of Qubit individuals

(v) Local exploitation before global exploration

\section{A. Sorting of items in input}

The items having a greater profit by weight ratio are considered to have higher probability of their inclusion in the optimal solution. Thus the items in input are sorted in the decreasing order of their profit by weight ratio. This sorting is used to initialize qubit individuals so that they can generate better solutions and also to improve repair procedure so that it provides better solutions.

Initializing the Qubit Individuals to depict the better estimations of distribution models: In order to initialize the qubit individuals, the items are divided in to 3 classes based on where they lie in order of preference; the first class contains items having high preference for selection in a knapsack, items in second part have intermediate preference and third contain items having low preference. Hence, qubits for items lying in first class (third class) are assigned values closer to $1(0)$ so that they have high (low) probability of collapsing to value 1 . Items lying in the second class require more processing for convergence to either 0 or 1 , hence intermed iate values between 0 and 1 are assigned to them.

As a result, QIEA-MKP starts exploiting the area or region in solution space having higher probability of having solutions closer to optimal. 


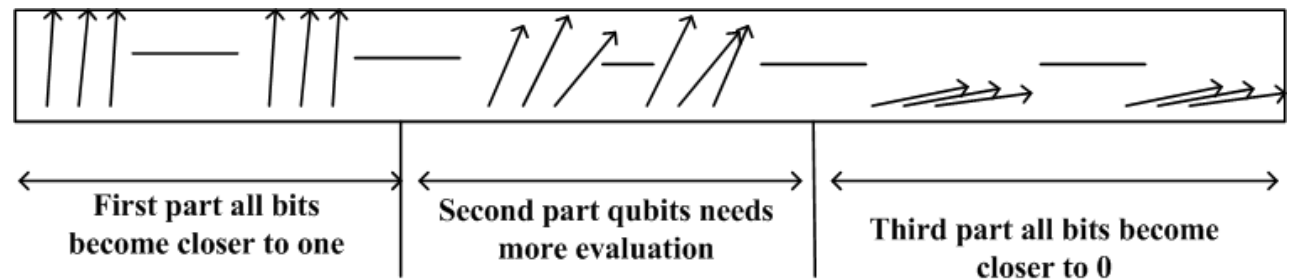

Fig. 2. Initialization of qubits. Qubits for items shown are sorted in decreasing profit by weight ratio from left to right

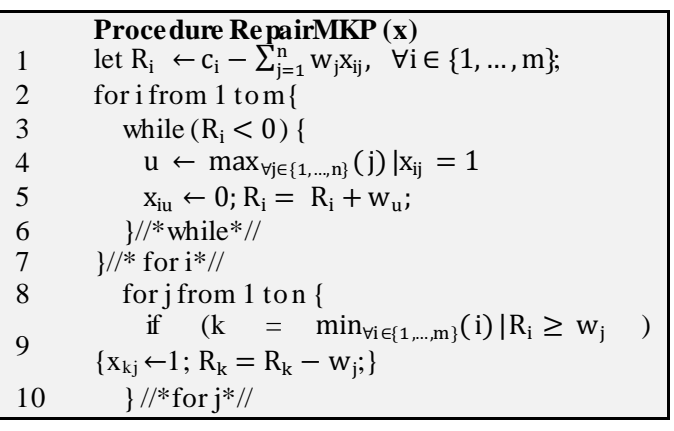

Fig. 3. Pseudo-code for RepairMKP

Modifying the repair function to improve quality of solutions: QIEA uses a simple "repair" function after it observes the qubits through the "make" procedure to make the observed solution feasible. In QIEA-MKP, the repair function is modified to improve the quality of solutions while making them feasible based on the phase 1 of MTHM heuristic presented in section IV. This improves the speed of convergence. As explained earlier the items are sorted in order of their preference to include them into a knapsack. So, in each repair step, items closest to the end are removed and items closest to the beginning are added as necessary. The knapsacks are assumed to be sorted in order of their increasing capacity and that's the order in which they are considered when items are added into knapsacks. The pseudo-code is given in Fig. 3.

\section{B. Improving the local best solutions}

The local best solutions are further improved in two stages based on the phases 1 and 2 of MTHM heuristic [29] described in section IV. In first stage it tries to exchange every pair of items assigned to different knapsacks along with inserting a new item so that total profit is increased. Secondly, every selected item (starting from last in the sorted order) is tried to be replaced by one of the remaining items so that the total profit sum is increased. The pseudo-code of these procedures is given in Fig. 4. and Fig. 5.

\section{Mutation of solutions appearing to be stuck in local optimum.}

EAs suffer from tendency of getting stuck in local optima. All the modifications described above help the algorith $m$ to exploit the search space around the greedy solutions increasing the speed of convergence but they also increase the tendency to get stuck in local optima. To combat this problem, if a new solution generated is seen to be close to global best solution found so far it is mutated. During mutation, after 2-3 bits in the solution vector are randomly selected and changed to 0 , the partial solution is improved using ImroveStage1. To check closeness of two solutions, Hamming distance between them is calculated. Such an operator improves diversity without increasing the computational effort. It helps to explore the solution space around a current solution such that local optimal in vicinity is not missed. This improves the chances of finding optimal in case it is in vicinity of the converging solution.

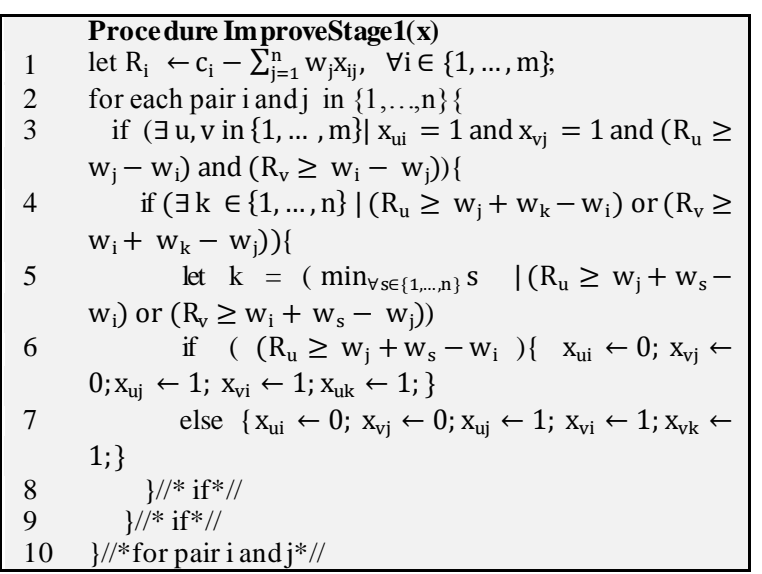

Fig. 4. Pseudo-code for ImproveStage1

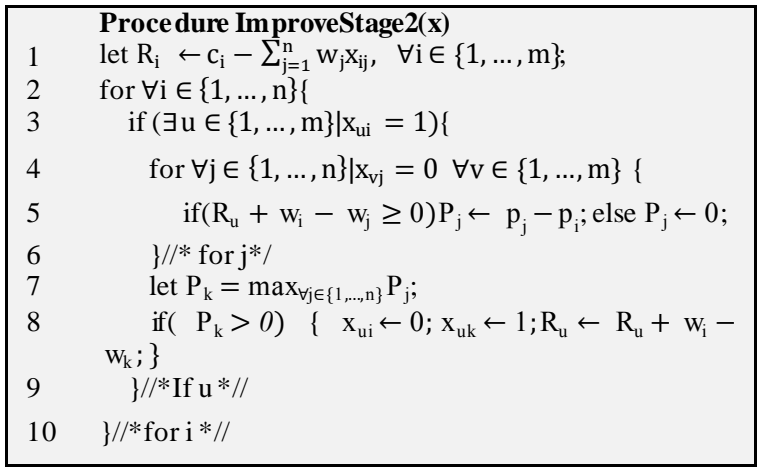

Fig. 5. Pseudo-code for ImproveStage2

\section{Re-initialization of Qubit individuals.}

It may happen even after applying mutation as explained in section 5.3 that all the solutions generated from a qubit individual are still same after a sequence of generations. It clearly indicates that such a qubit individual has converged and no further new solutions can be generated using them. Thus, each qubit in individuals which generate same solution for more that 3 times out of 5 is reset as explained in section 5.1. It 
increases the diversity of solutions explored through the qubit individuals without increasing the computational effort.

\section{E. Local exploitation before global exploration.}

QIEA has the property that it updates the qubits over the time period such that they represent Estimation of Distribution models. Thus, basic steps of QIEA are executed for some iterations to update some of the qubit individuals in itialized as described in section V.A.1. The steps listed in the following are performed on half of the qubit individuals for small number of times (empirically set as 15 for th is work) before starting QIEA-MKP on the entire population.

- Make

- RepairMKP

- ImproveStage 1

- ImproveStage 2

- Update

The execution of these steps of QIEA will also exploit intensively the area represented by the current qubit individuals before starting the QIEA-MKP which is balanced with respect to its power of exploitation and exploration. The resulting qubit individuals thus favour the small solution subspace close to better solutions.

A solution to MKP must specify whether the item is included or not and if it is included then the index of knapsack it has been put into. Hence an optimal solution does not require only the selection of correct items but also that they are packed into the correct knapsacks. In this work, two components (in the form of two binary strings) are used to represent a solution individual where, first of length $\mathrm{n}$ contains 0 or 1 for each item conveying about its selection status, and second of length $\left(\mathrm{n} * \log _{2} \mathrm{~m}\right)$ contains the index in binary of knapsack in which it is packed. The integers in $\{1, \ldots, m\}$ are represented by a bit string of length $\log _{2} \mathrm{~m}$. A qubit individual is thus represented by two components of lengths $n$ and $\mathrm{n} * \log _{2} \mathrm{~m}$ correspondingly.

The complete pseudo-code for QIEA-MKP is presented in Fig. 6. In the pseudo-code for MKP; $t$ refers to the current iteration, two components of population of qubit individuals after $t^{\text {th }}$ iteration are represented using $\mathrm{Q} 1(\mathrm{t})$ and $\mathrm{Q} 2(\mathrm{t}), \mathrm{P} 1(\mathrm{t})$ and $\mathrm{P} 2(\mathrm{t})$ represent the population of individual solutions, B1(t) and B2(t) is the set of best solutions corresponding to each individual, $\mathrm{c}_{i}$ is the capacity of the $\mathrm{i}^{\text {th }}$ knapsack. Individuals represented by $\mathrm{Q} 1(\mathrm{t})$ and $\mathrm{Q} 2(\mathrm{t})$ are referred to as $\mathrm{q}_{\mathrm{j}}^{\mathrm{t}}$ (composed of $\mathrm{q} \mathrm{1}_{\mathrm{j}}^{\mathrm{t}}$ and $\mathrm{q} 2_{\mathrm{j}}^{\mathrm{t}}$ ), individuals in $\mathrm{P} 1(\mathrm{t})$ and $\mathrm{P} 2(\mathrm{t})$ are referred to as $\mathrm{p}_{\mathrm{j}}^{\mathrm{t}}$ (composed of $\mathrm{p} 1_{\mathrm{j}}^{\mathrm{t}}$ and $\mathrm{p} 2_{\mathrm{j}}^{\mathrm{t}}$ ), similarly individuals in $\mathrm{B} 1(\mathrm{t})$ and $\mathrm{B} 2(\mathrm{t})$ are referred to as $b_{j}^{t}$ (composed of $b 1_{j}^{t}$ and $b 2_{j}^{t}$ ) for each $j \in n ; b$ refers to global best solution having components viz., b1 and b2. In the following paragraph a brief description is given for procedures called in QIEA-MKP but have not been described yet.
Initialize Greedy $\left(q 1_{j}^{t}\right)$ : where $q 1_{j}^{t}$ is $j^{\text {th }}$ qubit individual in a population $\mathrm{Q} 1(\mathrm{t})$. The procedure initializes the qubit individual $\mathrm{q}_{\mathrm{j}}^{\mathrm{t}}$ as explained in section V.A.

Make P1(t) and P2(t) from Q1(t) and Q2(t): The procedure collapses the qubit individuals in $\mathrm{Q} 1(\mathrm{t})$ (or $\mathrm{Q} 2(\mathrm{t})$ ) observing solution individuals in $\mathrm{P} 1(\mathrm{t})$ (or P2(t)).

HamDistance $\left(p 1_{j}^{s}, b 1\right)$ : Returns hamming distance between two binary strings.

$\operatorname{Mutate}\left(\mathrm{p} 1_{\mathrm{j}}^{\mathrm{s}}\right)$ : Mutates the solution $\mathrm{p} 1_{\mathrm{j}}^{\mathrm{s}}$ as described in section V.D.

Update $q_{j}^{t}$ based on $b_{j}^{t}$ : Rotates the qubits $q 1_{j}^{t}$ towards bits in $b 2_{j}^{t}$ and $q 2_{j}^{t}$ towards bits in $b 1_{j}^{t}$ as explained earlier and defined in [17] using the rotation angle as 0.01 .

Evaluate $\mathrm{P}(\mathrm{s})$ : Assign individuals in $\mathrm{P}(\mathrm{s})$ to their profit values.

The maximum number of iterations in algorithm is controlled using a global constant, Maxiterations.

\section{RESULTS AND DISCUSSION}

The experiments are done on Intel ${ }^{\circledR}$ Xeon ${ }^{\circledR}$ Processor E5645 ( 12M Cache, $2.40 \mathrm{GHz}, 5.86$ GT/s Intel® QPI ). The machine uses Red Hat Linux Enterprise 6.

The solutions converged for most of the problem instances considered here within 10 iterations hence maxIterations is set to 10 . Empirically, $\eta_{1}$ and $\eta_{2}$ are set to 5 and population size is set to 10 .

The experiments are performed to observe the effect of the modifications presented in sections V.A through V.E on basic QIEA framework discussed in the beginning of section V. The performance of QIEA and QIEA-MKP is observed on randomly generated instances having elements 1000, 5000 and 10000 with number of knapsacks as 2, 5 and 10.

The problem instances are randomly generated, using the generator of instances available at the Pisinger's home page viz. http://www.diku.dk/ pis inger/codes.html, where weights $w_{j}$ are distributed in $[1, R]$ and profits $p_{j}$ are calculated as $p_{j}=w_{j}+R / 10$. Such instances correspond to a real-life situation where the return is proportional to the investment plus some fixed charge for each project.

Two different classes of capacities are considered for the knapsacks in randomly generated instances viz., similar and dissimilar. In instances with similar capacities the first $\mathrm{m}-1$ capacities $\mathrm{c}_{\mathrm{i}}, \mathrm{i} \square\{1, \ldots, \mathrm{m}-1\}$ are distributed in

$$
\left[0.4 \sum_{\mathrm{j}=1}^{\mathrm{n}} \mathrm{w}_{\mathrm{j}} / \mathrm{m}, 0.6 \sum_{\mathrm{j}=1}^{\mathrm{n}} \mathrm{w}_{\mathrm{j}} / \mathrm{m}\right] \square \mathrm{i} \square\{1, \ldots, \mathrm{m}-1\}
$$

while instances with dissimilar capacities have these $c_{i}$ distributed in

$$
\left[0,0.5\left(\sum_{\mathrm{j}=1}^{\mathrm{n}} \mathrm{w}_{\mathrm{j}}-\sum_{\mathrm{k}=1}^{\mathrm{i}-1} \mathrm{c}_{\mathrm{k}}\right)\right] \text { for } \mathrm{i}=1, \ldots, \mathrm{m}-1
$$

The last capacity $\mathrm{c}_{\mathrm{m}}$ in both classes is chosen as

$$
\mathrm{c}_{\mathrm{m}}=0.5 \sum_{\mathrm{j}=1}^{\mathrm{n}} \mathrm{w}_{\mathrm{j}}-\sum_{\mathrm{i}=1}^{\mathrm{m}-1} \mathrm{c}_{\mathrm{i}}
$$


Tables 2 and 3 present the performance of QIEA and QIEA-MKP for the instances having similar capacities. Tables 4 and 5 present the performance of QIEA and QIEA-MKP for the instances having dissimilar capacities. Instances are randomly generated with number of elements ranging from 1000 to 10000 and number of knapsacks ranging from 2 to 100 . The instances having 100 knapsacks of dissimilar capacities could not be generated.

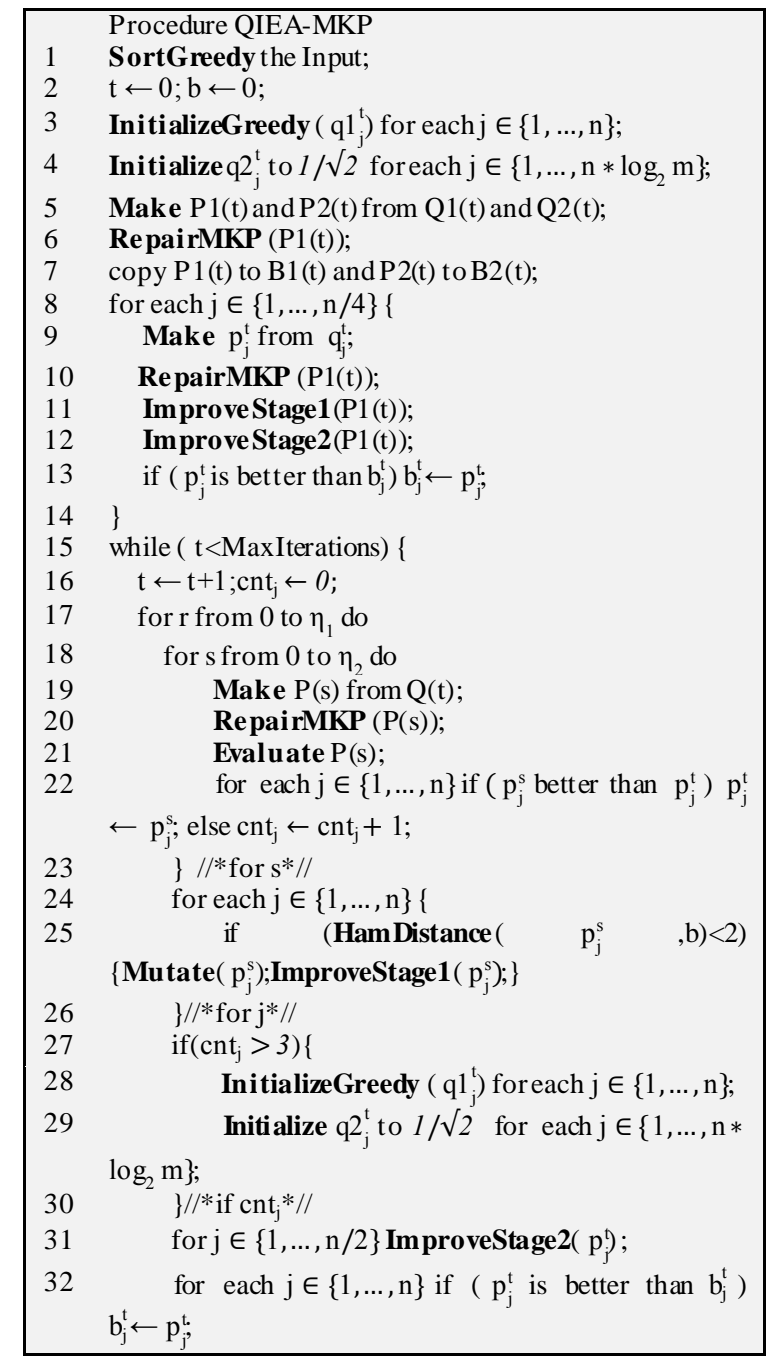

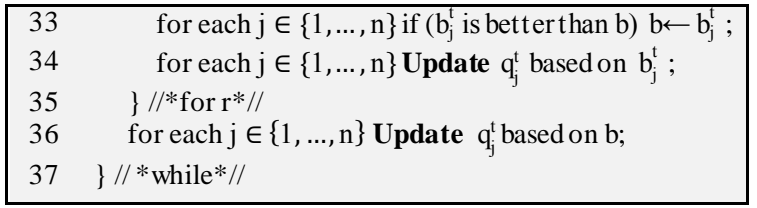

Fig. 6. Pseudo-code for QIEA-MKP

The tables show a comparison of profit values obtained using the specific format of QIEA with values obtained using the heuristic mentioned in section IV. The best, average, worst, standard deviation in profit values obtained within 30 independent runs of the algorithm have been reported along with the minimum and average computaional effort required in terms of number of function evaluations (FES) as to reach the best (MinFES and AvgFES respectively) and RDH relative distance of best solution from heuristic. If profit for the instances obtained using exact algorithm is $\mathrm{P}_{\mathrm{h}}$ and best profit obtained is $\mathrm{P}_{b}$ the $\mathrm{RDH}$ is calculated as follows.

$$
\mathrm{RDH}=\left(\left(\mathrm{P}_{\mathrm{b}}-\mathrm{P}_{\mathrm{h}}\right) / \mathrm{P}_{\mathrm{h}}\right) * 100
$$

The convergence of profit values achieved using QIEA and QIEA-MKP is studied and compared. Fig 7 shows how the best profit value obtained through QIEA and QIEA-MKP converges. The iteration number and the profit achieved have been plotted on $\mathrm{x}$-axis and $\mathrm{y}$-axis respectively. QIEA-MKP executes the basic steps of QIEA for 15 times during initialization of half of the qubit individuals as explained in section V.E. Results for these additional steps are plotted using iteration numbers from I0 to I15 on x-axis, where values exist only for QIEA-MKP.

It is clear from fig 7 that

- Both algorithms start with similar profits.

- QIEA-MKP forgoes ahead of QIEA during initialization steps.

- However, QIEA-MKP finds significantly better solutions with in a few iterations of the main loop of the algorithm.

- Even after 50 iterations of the main loop, QIEA is still far away from the value achieved using QIEA-MKP in a few iterations

Table 2. Performance of QIEA on instances with similar capacities

\begin{tabular}{|cc|cccccccc|}
\hline $\mathrm{n}$ & $\mathrm{m}$ & Heuristic (MTHM) & Best & Average & Worst & Stddev & MinFES & AvgFES & RDH \\
\hline \multirow{4}{*}{1000} & 2 & 258382 & 256590 & 256523.7 & 256502 & 17.36852 & 306 & 2375.17 & -0.6935 \\
& 5 & 258192 & 256549 & 256524.7 & 256503 & 11.00763 & 584 & 2405.53 & -0.6363 \\
& 10 & 257594 & 256610 & 256576.7 & 256556 & 12.89302 & 1867 & 2702.2 & -0.3820 \\
& 100 & 238909 & 256157 & 256057.4 & 255992 & 38.94619 & 15 & 631.73 & 7.2195 \\
\hline \multirow{4}{*}{5000} & 2 & 1311752 & 1302242 & 1302143 & 1302100 & 32.39831 & 110 & 1775.07 & -0.7250 \\
& 5 & 1311602 & 1302117 & 1302070 & 1302036 & 23.06684 & 86 & 1286.67 & -0.7232 \\
& 10 & 1310793 & 1302151 & 1302094 & 1302059 & 19.87617 & 162 & 1912.03 & -0.6593 \\
& 100 & 1295826 & 1302426 & 1302353 & 1302316 & 26.17703 & 1571 & 2648.83 & 0.5093 \\
\hline \multirow{4}{*}{10000} & 2 & 2607179 & 2587373 & 2587291 & 2587187 & 48.8818 & 19 & 858.4 & -0.7597 \\
& 5 & 2606773 & 2587174 & 2587092 & 2587012 & 47.10786 & 48 & 994.9 & -0.7518 \\
& 10 & 2606811 & 2587191 & 2587090 & 2587020 & 42.17266 & 1 & 872.23 & -0.7526 \\
& 100 & 2591142 & 2587730 & 2587459 & 2587371 & 76.72193 & 1743 & 2643.23 & -0.1317 \\
\hline
\end{tabular}


Table 3. Performance of QIEA-MKP on instances with Similar Capacities

\begin{tabular}{|cc|cccccccc|}
\hline $\mathrm{n}$ & $\mathrm{m}$ & Heuristic (MTHM) & Best & Average & Worst & Stddev & MinFES & AvgFES & RDH \\
\hline \multirow{4}{*}{1000} & 2 & 258382 & 258379 & 258370.7 & 258363 & 4.648569 & 19 & 27.4 & -0.0012 \\
& 5 & 258192 & 258384 & 258376.1 & 258363 & 5.030482 & 4 & 28.1 & 0.0744 \\
& 10 & 257594 & 258373 & 258352.7 & 258306 & 17.00943 & 7 & 25.57 & 0.3024 \\
& 100 & 238909 & 258158 & 257781 & 257207 & 233.6354 & 1 & 15.47 & 8.0570 \\
\hline \multirow{4}{*}{5000} & 2 & 1311752 & 1311764 & 1311735 & 1311704 & 14.21445 & 21 & 30.7 & 0.0009 \\
& 5 & 1311602 & 1311814 & 1311797 & 1311783 & 8.027324 & 21 & 29.83 & 0.0162 \\
& 10 & 1310793 & 1311810 & 1311773 & 1311713 & 21.78252 & 20 & 30.53 & 0.0776 \\
& 100 & 1295826 & 1311638 & 1311506 & 1311387 & 58.71624 & 1 & 16.3 & 1.2202 \\
\hline \multirow{4}{*}{10000} & 2 & 2607179 & 2607091 & 2607052 & 2606995 & 26.76444 & 24 & 31 & -0.0034 \\
& 5 & 2606773 & 2607224 & 2607188 & 2607155 & 21.01031 & 21 & 32.7 & 0.0173 \\
& 10 & 2606811 & 2607225 & 2607192 & 2607173 & 19.30918 & 20 & 30.2 & 0.0159 \\
& 100 & 2591142 & 2606844 & 2606714 & 2606555 & 66.10837 & 1 & 15.63 & 0.6060 \\
\hline
\end{tabular}

Table 4. Performance of QIEA on instances with dissimilar capacities

\begin{tabular}{|cc|cccccccc|}
\hline $\mathrm{n}$ & $\mathrm{m}$ & Heuristic (MTHM) & Best & Average & Worst & Stddev & MinFES & AvgFES & RDH \\
\hline \multirow{3}{*}{1000} & 2 & 258367 & 256520 & 256492.8 & 256476 & 10.61451 & 295 & 2363.367 & -0.7149 \\
& 5 & 257915 & 256562 & 256527.1 & 256499 & 15.35185 & 405 & 2506.3 & -0.5246 \\
& 10 & 258078 & 256560 & 256528.4 & 256509 & 14.1067 & 813 & 2620.933 & -0.5882 \\
\hline \multirow{3}{*}{5000} & 2 & 1311854 & 1302136 & 1302057 & 1302025 & 22.84641 & 69 & 1243.367 & -0.7408 \\
& 5 & 1311710 & 1302115 & 1302059 & 1302012 & 24.81018 & 109 & 1506.233 & -0.7315 \\
& 10 & 1311663 & 1302055 & 1302016 & 1301987 & 16.4835 & 42 & 2526.633 & -0.7325 \\
\hline \multirow{3}{*}{10000} & 2 & 2607147 & 2587170 & 2587070 & 2587006 & 44.68634 & 12 & 713.6667 & -0.7662 \\
& 5 & 2606951 & 2587346 & 2587245 & 2587166 & 49.82521 & 73 & 580.5667 & -0.7520 \\
& 10 & 2606167 & 2587387 & 2587207 & 2587137 & 58.89777 & 8 & 719.3333 & -0.7206 \\
\hline
\end{tabular}

Table 5. Performance of QIEA-MKP on instances with dissimilar capacities

\begin{tabular}{|cc|cccccccc|}
\hline $\mathrm{n}$ & $\mathrm{m}$ & Heuristic $($ MTHM) & Best & Average & Worst & Stddev & MinFES & AvgFES & RDH \\
\hline \multirow{3}{*}{1000} & 2 & 258367 & 258384 & 258378.9 & 258374 & 4.232333 & 9 & 27.7 & 0.0066 \\
& 5 & 257915 & 258383 & 258369.2 & 258358 & 5.628744 & 19 & 30.37 & 0.1815 \\
& 10 & 258078 & 258362 & 258345.5 & 258328 & 7.69124 & 14 & 27.5 & 0.1100 \\
\hline \multirow{3}{*}{5000} & 2 & 1311854 & 1311824 & 1311806 & 1311794 & 7.764419 & 20 & 30.2 & -0.0023 \\
& 5 & 1311710 & 1311796 & 1311776 & 1311754 & 10.88545 & 21 & 31.3 & 0.0066 \\
& 10 & 1311663 & 1311824 & 1311795 & 1311768 & 14.31035 & 16 & 30.2 & 0.0123 \\
\hline \multirow{3}{*}{10000} & 2 & 2607147 & 2607235 & 2607211 & 2607187 & 13.51496 & 22 & 30.83 & 0.0034 \\
& 5 & 2606951 & 2607165 & 2607114 & 2607067 & 21.29422 & 23 & 31.7 & 0.0082 \\
& 10 & 2606167 & 2607191 & 2607144 & 2607065 & 26.99104 & 21 & 30.3 & 0.0393 \\
\hline
\end{tabular}

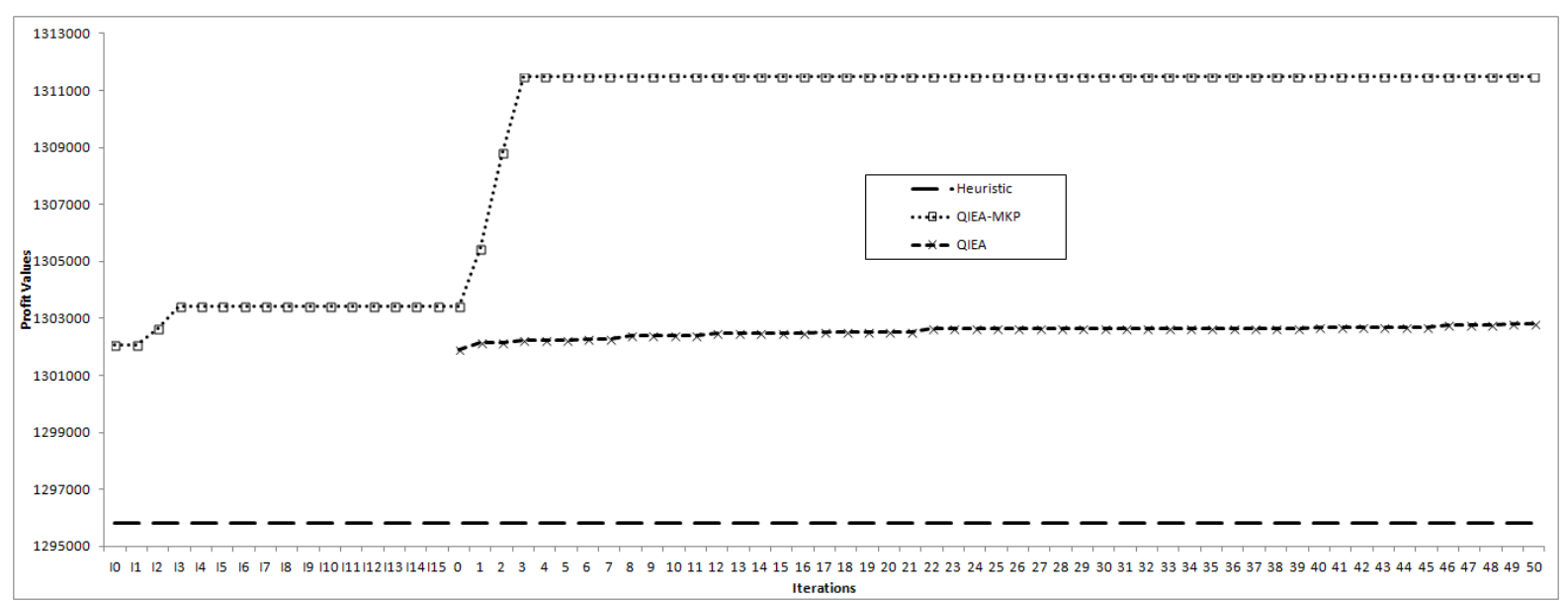

Fig. 7. Comparing convergence of best value achieved using QIEA and QIEA-MKP (an instance having $\mathrm{n}=5000, \mathrm{~m}=100$ ) 


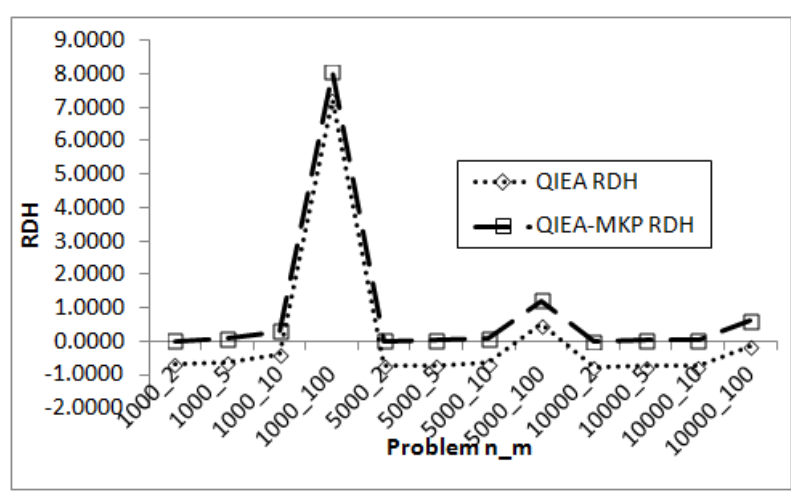

Instances with Similar Capacities

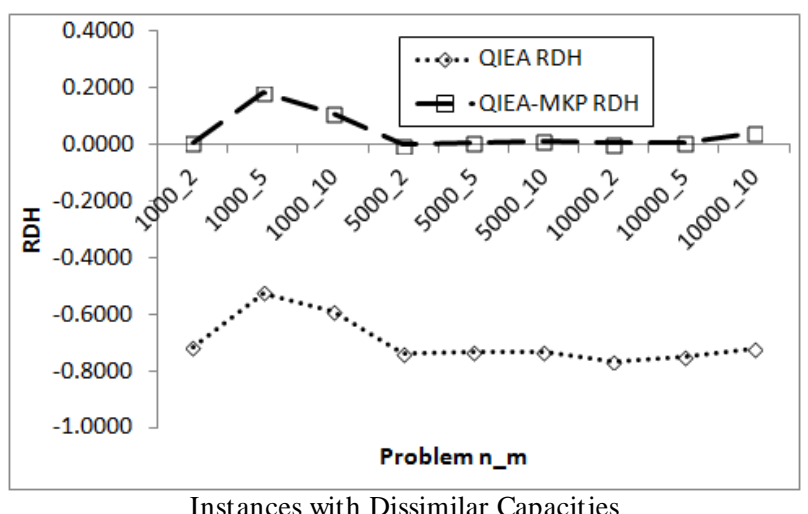

Fig. 8. Comparison of QIEA and QIEA-MKP on the basis of RDH

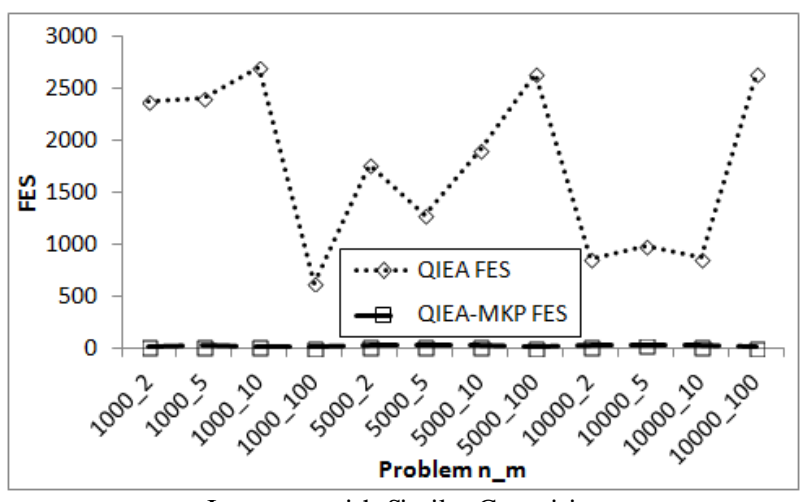

Instances with Similar Capacities

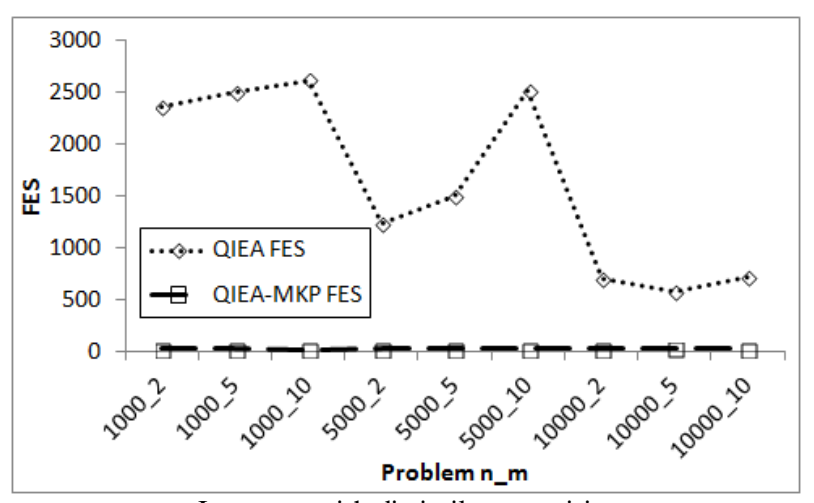

Instances with dissimilar capacities

Fig. 9. Comparison of QIEA and QIEA-MKP on the basis of FES required reaching their best solution.

Figures 8 and 9 present a comparison of QIEA and QIEA-MKP on the basis of quality of solutions and computational effort. RDH of the solutions obtained using QIEA and QIEA-MKP with respect to size of problem instances having different classes of capacities considered is shown in fig 8 . Fig 9 plots the Average FES required using QIEA and QIEA-MKP with respect to size of problem instances having different classes of capacities considered.

Following points are observed from the results:

- QIEA-MKP shows considerable improvement both in quality of solutions obtained and computational effort required to reach the best as compared to QIEA.

- The solutions obtained from QIEA-MKP are much better than the heuristic used to improve solutions locally for most of the instances tested. This improvement is better for instances which required to fill more number of knapsacks.

- The hybridised QIEA-MKP is able to provide much better solutions within very less number of FES as compared to QIEA used as the base.

- The graph showing quality of solutions with respect to heuristic solution (i.e. RDH) for QIEA-MKP is similar in shape as of QIEA but with a shift from area having solution worse than heuristic to the area having better solution than heuristic.

\section{CONCLUSIONS}

The QIEA is improved by embedding within it the local improvement based on a known effective heuristic for MKP with an objective to imp rove exploitation. Apart from it some techniques viz. mutation of solutions appearing to be close to local optima, and reinitializing qubit individuals found incapable to generate new solutions are also induced in order to improve the power to explore the search space. This way the proposed QIEA, with balanced power of exploitation and exploration of search space, provide significantly better solutions with respect to both the QIEA used as base and the heuristic used for proposed hybridization. The proposed QIEA provide better results using much reduced computational effort than basic QIEA.

\section{ACKNOWLEDGMENTS}

Authors are grateful to Department of Science and Technology, India (DST) and Deutsche Forschungs gemeinschaft, Germany (DFG) for the support under project No. INT/FRG/DFG/P-38/2012 titled "A lgorithm Engineering of Quantum Evolutionary Algorithms for Hard Optimization Problems".

\section{REFERENCES}

[1] C. Chekuri and S. Khanna, "A PTAS for the multiple knapsack problem," SIAM Journal on Computing, vol. 35, pp. 713-728, 2006. 
[2] S.Y. Yang, M. Wang, and L.C. Jiao, "A novel quantum evolutionary algorithm and its application," in Proc CEC, 2004, pp. 820-826.

[3] C. Patvardhan, Apurva Narayan, and A. Srivastav, "Enhanced Quantum Evolutionary Algorithms for Difficult Knapsack Problems," in PReMI'07 Proceedings of the 2nd international conference on Pattern recognition and machine intelligence, 2007, pp. 252-260.

[4] M. D. Platel, S. Schliebs, and N. Kasabov, "A versatile quantum-inspired evolutionary algorithm," in Proc. CEC, 2007, pp. 423-430.

[5] G. S. Sailesh Babu, D. Bhagwan Das, and C. Patvardhan, "Real-Parameter quantum evolutionary algorithm for economic load dispatch," IET Gener. Transm. Distrib., vol. 2, no. 1, pp. 22-31, 2008.

[6] A. Mani and C. Patvardhan, "A Hybrid quantum evolutionary algorithm for solving engineering optimization problems," International Journal of Hybrid Intelligent Systems, vol. 7, pp. 225-235, 2010.

[7] Ling Wang and Ling-po Li, "An effective hybrid quantuminspired evolutionary algorithm for parameter estimation of chaotic systems," Expert Systems with Applications, vol. 37, no. 2, pp. 1279-1285, 2010.

[8] Shuyuan Yang, Min Wang, and Licheng Jiao, "Quantuminspired immune clone algorithm and multiscale Bandelet based image representation," Pattern Recognition Letters, vol. 31, no. 13, pp. 1894-1902, 2010.

[9] Jing Xiao, YuPing Yan, Jun Zhang, and Yong Tang, "A quantum-inspired genetic algorithm for k-means clustering," Expert Systems with Applications, vol. 37, no. 7, pp. 4966-4973, 2010.

[10] P. Arpaia, D. Maisto, and C. Manna, "A Quantum-inspired Evolutionary Algorithm with a competitive variation operator for Multiple-Fault Diagnosis," Applied Soft Computing, vol. 11, no. 8, pp. 4655-4666, 2011.

[11] C Patvardhan, P Prakash, and A Srivastav, "A novel quantum-inspired evolutionary algorithm for the quadratic knapsack problem," Int. J. Mathematics in Operational Research, vol. 4, no. 2, pp. 114-127, 2012.

[12] K. Han and J. Kim, "On setting the parameters of quantum-inspired evolutionary algorithm for practical application.," in Proc. CEC, 2003, pp. 178-184.

[13] K-H Han, "On the Analysis of the Quantum-inspired Evolutionary Algorithm with a Single Individual," in IEEE Congress on Evolutionary Computation, Vancouver, Canada, 2006.

[14] M. D. Platel, Stefan Schliebs, and Nikola Kasabov, "Quantum-Inspired Evolutionary Algorithm: A Multimodel EDA," IEEE Transactions on Evolutionary Computation, vol. 13, no. 6, pp. 1218-1232, 2009.

[15] Gexiang Zhang, "Quantum-inspired evolutionary algorithms: a survey and empirical study," Journal of Heuristics, vol. 17, no. 3, pp. 303-351, 2011.

[16] C. Blum, J. Puchinger, G. R. Raidl, and A. Roli, "Hybrid metaheuristics in combinatorial optimization: A survey," Applied Soft Computing, vol. 11, pp.4135-4151, 2011.

[17] Kuk-Hyun Han and Jong-Hwan Kim, "Quantum-Inspired Evolutionary Algorithm for a Class of Combinatorial Optimization," IEEE Transactions on Evolutionary Computation, vol. 6, no. 6, pp. 580-593, December 2002.

[18] K. Han and J. Kim, "Quantum-inspired evolutionary algorithms with a new termination criterion, h-epsilon gate, and two-phase scheme.," IEEE Trans. Evol. Comput., vol. 8, no. 2, pp. 156-169, 2004.

[19] Zhongyu Zhao, Xiyuan Peng, Yu Peng, and Enzhe Yu, "An Effective Repair Procedure based on Quantuminspired Evolutionary Algorithm for 0/1 Knapsack
Problems," in Proceedings of the 5th WSEAS Int. Conf. on Instrumentation, Measurement, Circuits and Systems, Hangzhou, 2006, pp. 203-206.

[20] G.X. Zhang, M. Gheorghe, and C.Z. Wu, "A quantuminspired evolutionary algorithm based on $\mathrm{p}$ systems for knapsack problem," Fund. Inf., vol. 87, no. 1, pp. 93-116, 2008.

[21] M. -H. Tay arani-N and M. -R. Akbarzadeh-T, "A Sinusoid Size Ring Structure Quantum Evolutionary Algorithm," in IEEE Conference on Cybernetics and Intelligent Systems, 2008, pp. $1165-1170$.

[22] Zhiy ong Li, Günter Rudolph, and Kenli Li, "Convergence performance comparison of quantum-inspired multiobjective evolutionary algorithms," Computers and Mathematics with Applications, vol. 57, pp. 1843-1854, 2009.

[23] Parvaz Mahdabi, Saeed Jalili, and Mahdi Abadi, "A multistart quantum-inspired evolutionary algorithm for solving combinatorial optimization problems," in (GECCO '08) Proceedings of the 10th annual conference on Genetic and evolutionary computation, 2008, pp. 613-614.

[24] Takahiro Imabeppu, Shigeru Nakayama, and Satoshi Ono, "A study on a quantum-inspired evolutionary algorithm based on pair swap," Artif Life Robotics, vol. 12, pp. 148$152,2008$.

[25] T-C Lu and G-R Yu, "An adaptive population multiobjective quantum-inspired evolutionary algorithm for multi-objective 0/1 knapsack problems," Information Sciences, vol. 243, pp. 39-56, 2013.

[26] Y. Kim, J. H. Kim, and K. H. Han, "Quantum-inspired multiobjective evolutionary algorithm for multiobjective 0/1 knapsack problems," in Proc. CEC, 2006, pp. 26012606.

[27] K. Han, K. Park, C. Lee, and J. Kim, "Parallel quantuminspired genetic algorithm for combinatorial optimization prblem," in Proc. CEC, vol. 2, 2001, pp. 1422-1429.

[28] R. Nowotniak and J. Kucharski, "GPU-based tuning of quantum-inspired genetic algorithm for a combinatorial optimization problem," BULLETIN OF THE POLISH ACADEMY OF SCIENCES: TECHNICAL SCIENCES, vol. 60, no. 2, pp. 323-330, 2012.

[29] S. Martello and P. Toth, "Solution of the zero-one multiple knapsack problem," European Journal of Operational Research, vol. 4, no. 4, pp. 276-283, 1980.

[30] F. Diedrich and K. Jansen, "Improved approximation algorithms for scheduling with fixed jobs," in Proceedings of the 20th ACM-SIAM Symposium on Discrete Algorithms (SODA2009), 2009, pp. 675-684.

[31] S. Eilon and N. Christofides, "The loading problem," Management Science, vol. 17, pp. 259-268, 1971.

[32] Hans Kellerer, Ulrich Pferschy, and David Pisinger, Knapsack Problems. Berlin.Hiedelberg: Springer-Verlag, 2004.

[33] M. S. Hung and J. C. Fisk, "An algorithm for the 0-1 multiple knapsack problems," Naval Reserach Logistics Quarterly, vol. 24, pp.571-579, 1978.

[34] S. Martello and P. Toth, "A bound and bound algorithm for the zero-one," Discrete Applied Mathematics, vol. 3, pp. 275-288, 1981.

[35] H. Kellerer, "A polynomial time approximation scheme for the multiple knapsack problem," in Proceedings of the 2nd Workshop on Approximation Algorithms for Combinatorial Optimization Problems, APPROX(1999), LNCS 1671, 1999, pp. 51-62.

[36] K. Jansen, "Parameterized approximation scheme for the multiple knapsack problem," Siam Journal on Computing, vol. 39, no. 4, pp. 665-674, 2009. 
[37] K. Jansen, "A fast approximation scheme for the multiple knapsack problem," in 38th Conference on Current Trends in Theory and Practice of Computer Science, LNCS 7147, 2012, pp. 313-324.

[38] S. Martello and P. Toth, Knapsack Problems: Algorithms and Computer Implementations. Chichester, UK: Wiley, 1990.

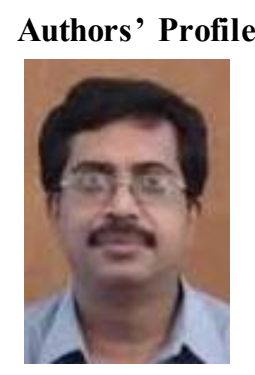

C. Patvardhan is working in the Day albagh Educational Institute, Agra as Professor in Electrical Engineering and Coordinator of the PG program in Faculty of Engineering, DEI. He obtained his BSc (Engg.) degree from Dayalbagh in 1987, MTech from IISc, Bangalore in Computer Science in 1989 and his $\mathrm{PhD}$ from DEI in 1994. He has published more than 250 papers in journals and proceedings of conferences and has won 20 Best Paper Awards. He has also published one book and has been an Editor of three Conference proceedings. $\mathrm{He}$ has been the Investigator/Co-Investigator of several funded $\mathrm{R} \& \mathrm{D}$ projects. He has been a consultant on Advanced Algorithms to various EDA industries. His current research interests are quantum and soft computing and image processing.

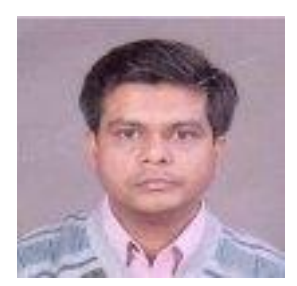

Sulabh Bansal is a Research Associate and Ph.D. scholar with the Department of Electrical Engineering, Dayalbagh Educational Institute, Agra. $\mathrm{He}$ obtained BE (Hons.)(Computer Science and Engineering) in 2000 and MTech in 2011. He has published several research papers in various national and international conferences and journals. He has rich 12 years experience diversified in software industry as developer and team leader and in academics as an educator and researcher. He has successfully managed several software development projects in the industry and guided several B.Tech. and M.Tech. level projects. His current research interests are evolutionary algorithms, optimization problems and parallel programming.

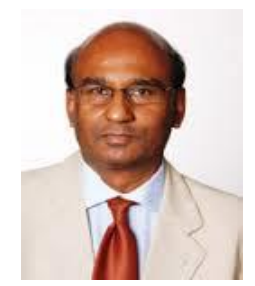

Anand Srivastav is a Professor in the Department of Computer Science, Christian-Albrechts-Universitat zu Kiel, Germany. He obtained his doctorate from the University of Munster in 1988. Thereafter, he worked as an Assistant Professor in the University of Bonn, the New York University, Yale University, the Free University, Berlin and Humboldt University of Berlin. He has held senior positions at several institutions, and has been director of the Computational Science Center at ChristianAlbrechts-Universitat zu Kiel since 2007. He was a speaker of the DFG Research Training Group, "Efficient Algorithms and Multiscale Methods" from 2000-2005, and has been the PI of the German Research Foundation's Future Ocean Cluster at Christian-Albrechts-Universitat since 2006. He is also the PI for the "Materials for Life" Cluster of Excellence proposed in 2010 at Christian-Albrechts-Universitat, Kiel.
How to cite this paper: C. Patvardhan, Sulabh Bansal, Anand Srivastav,"Balanced Quantum-Inspired Evolutionary Algorithm for Multiple Knapsack Problem", International Journal of Intelligent Systems and Applications(IJISA), vol.6, no.11, pp.111, 2014. DOI: $10.5815 /$ ijisa.2014.11.01 